\title{
Deformation of Pipelines Induced by the Construction of Underlying Twin-Tunnel
}

\author{
Jinlong ZHU*, Dayong ZHU
}

\begin{abstract}
The soil displacement load induced by the metro tunneling is applied to the adjacent pipelines, which leads to the deformation of the pipelines, and affects the safe use of the pipeline. According to the displacement of the soil caused by the excavation of the twin-tunnel, considering the shear deformation of the soil and the angle between the tunnel and the pipeline, the analysis method of the Pasternak foundation beam for the longitudinal displacement of the pipeline caused by tunneling was established. The calculation result was relatively close to that of the numerical simulation based on displacement controlled method (DCM) and the field measured data. The influences of the angle, the distance between tunnel axes, the soil volume loss rate and the relative bending stiffness on the pipeline deformation were analyzed. Finally, the influence of the shearing stiffness of the pipeline was researched based on the Timoshenko beam model.
\end{abstract}

Keywords: Pasternak foundation; pipeline; shearing stiffness; tunnel

\section{INTRODUCTION}

During the construction of urban subway, the tunnels crossing pipeline is normally inevitable. However, the displacement load of the upper soil caused by tunneling will be applied on the pipelines, resulting in deformation of the pipelines, which will seriously affect their normal and safe service. Attewell and other researchers [1] first analyzed the influence of tunnel excavation on the longitudinal deformation of the upper pipeline by using the two-stage method based on the Winkler elastic foundation beam model. This method is one of the commonly used methods to describe the pipe-soil interaction [2, 3]. However, the Winkler foundation model does not consider the continuity of foundation deformation and ignores the shearing deformation of soil. Some scholars adopted Parsnek foundation model in the two-stage method and obtained relatively accurate results $[4,5]$. A number of researches are confined to single line tunnel; actually the analysis of the influence of twin-tunnel construction on the deformation of the pipeline is of greater importance. Moreover, the researchers are generally concentrated on the condition of pipeline perpendicular to the tunnel. Shi and other researchers [6], based on the centrifuge model test, indicated that the angle size has a great influence on the pipeline settlement and strain caused by tunneling. Moreover, the influence of the pipeline shearing stiffness on the deformation is also one of the factors needed to be considered. In addition to the two-stage equivalent foundation beam method, some scholars have made helpful explorations on this problem based on the numerical simulation by the displacement control method [7, 8]. The displacement control method can directly apply displacement around the tunnel to reflect the stress release caused by excavation. It can simulate the soil loss rate of the stratum with high accuracy.

This paper considered the shearing stiffness of the foundation and the angle of the tunnel and pipeline, and established Pasternak foundation beam analysis method targeting at the pipeline longitudinal displacement caused by tunnel excavation, and compared the calculation results with DCM numerical simulation and field measurement based on a case, and analysed the influence of the angle, the tunnel axes distance, soil loss rate, relative bending stiffness on the pipeline deformation, and finally, the influence of pipeline shearing stiffness based on Timoshenko beam model was taken into account.

\section{SOIL DISPLACEMENT CAUSED BY TUNNEL EXCAVATION}

The settlement of the soil below the ground caused by tunneling still conforms to the Gaussian distribution law [9], and the settlement trough width is related to the tunnel depth and the ground settlement trough width coefficient [10], Combined with Liu's study about the twin-tunnel based on the superposition principle [11], the formula of the formation settlement induced by the twin-tunnel excavation is:

$$
\begin{aligned}
& S=\frac{1.25 R^{2} V_{1}}{K\left(z_{1}-z_{2}\right)} \cdot\left\{\exp \left[-\frac{\left(x-\frac{L}{2}\right)^{2}}{2 K^{2}\left(z_{1}-z_{2}\right)^{2}}\right]+\exp \left[-\frac{\left(x+\frac{L}{2}\right)^{2}}{2 K^{2}\left(z_{1}-z_{2}\right)^{2}}\right]\right\} \\
& K=\frac{1-\eta\left(\frac{z_{2}}{z_{1}}\right)}{1-\frac{z_{2}}{z_{1}}} K_{0}
\end{aligned}
$$

where $R$ is the tunnel radius $(m) ; V_{1}$ is the soil volume loss rates caused by single tunnel excavation; $z_{1}$ is the tunnel depth $(m) ; S$ is the settlement of the soil with $z_{2}$ in depth $(m), L$ is the distance of the twin-tunnel axes $(m)$; and $K_{0}$ is the settlement trough width coefficient of the ground. $K$ is the settlement trough width coefficient of the soil with $z_{2}$ in depth, and $\eta$ is the parameter about the soil, which is 0.65 for clay soil and 0.50 for sandy soil.

\section{PIPELINE DEFORMATION}

The two-stage foundation beam analysis method is used to study the settlement of the upper pipeline caused by tunnel excavation. It is assumed that the pipeline is always in contact with the soil. The pipeline is regarded as the Pasternak foundation beam, and the generalized displacement load caused by tunnel excavation is applied to the elastic foundation beam, and a balanced differential equation for the longitudinal deformation of the pipeline is 
established. The tunnel crosses the pipeline vertically or obliquely, as shown in Fig. 1, when the angle between the two is $\theta$, the displacement of the soil at the location of the pipeline caused by the twin-tunnel excavation is:

$f(x)=\frac{1.25 R^{2} V_{1}}{K\left(z_{1}-z_{2}\right)} \cdot\left\{\exp \left[-\frac{\left(x \sin \theta-\frac{L}{2}\right)^{2}}{2 K^{2}\left(z_{1}-z_{2}\right)^{2}}\right]+\exp \left[-\frac{\left(x \sin \theta+\frac{L}{2}\right)^{2}}{2 K^{2}\left(z_{1}-z_{2}\right)^{2}}\right]\right\}$

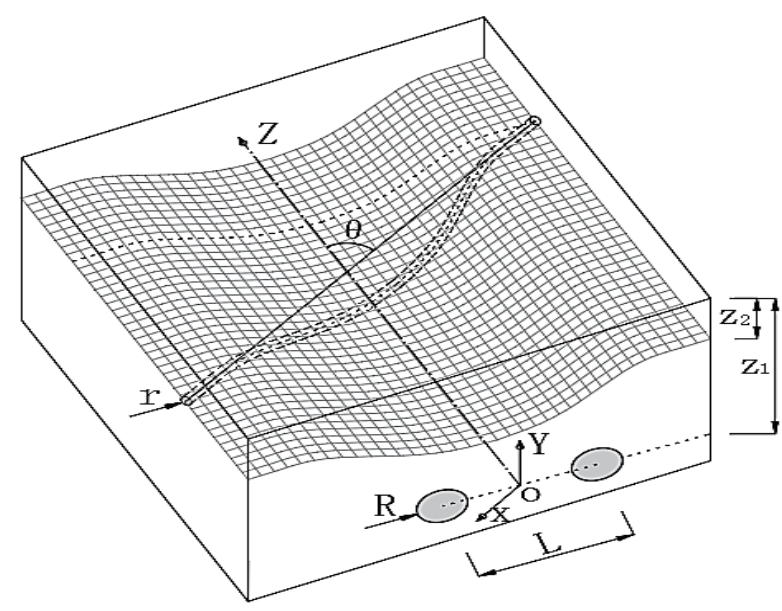

Figure 1 Calculation sketch

The two-parameter Pasternak foundation model contains the spring layer and the shear layer reflecting the soil properties, and the relationship between the ground reaction force and the soil layer displacement is:

$p(x)=-G \frac{\mathrm{d}^{2} f(x)}{\mathrm{d} x^{2}}+k f(x)$

where, $p(x)$ is the ground reaction force, $G$ is the foundation shear modulus $(\mathrm{N} / \mathrm{m})$, and $k$ is the foundation bed coefficient $(\mathrm{Pa} / \mathrm{m})$. Based on the simplified elastic space method $[12,13]$, for a foundation with an elastic modulus of $E(\mathrm{~Pa})$, the Poisson's ratio of $v$, and a thickness of $h$, there is:

$$
\begin{aligned}
& G=\frac{E H}{6(1+v)} \\
& k=\frac{E}{H}
\end{aligned}
$$

According to $\mathrm{Xu}$ Ling's research, the additional stress at the base where 6 times of pipeline diameter below the pipeline decreases into a negligible extent, so the $H$ value is taken as 6 times the pipeline diameter [14].

The pipeline is set as an Euler-Bernoulli beam, and the equilibrium differential equation of the pipeline with $z_{2}$ in depth is:

$$
\frac{\mathrm{d}^{4} w}{\mathrm{~d} x^{4}}-\frac{2 r G}{D} \frac{\mathrm{d}^{2} w}{\mathrm{~d} x^{2}}+\frac{2 r k}{D} w=\frac{2 r k}{D} f(x)-\frac{2 r G}{D} f(x)^{\prime \prime}
$$

where $w$ is the settlement of the pipeline, $r$ is the pipeline radius, and $D$ is the bending stiffness of the pipeline.
Tunnel excavation does not affect the pipeline in the distance, and there is:

$$
\begin{aligned}
& \left.w(x)\right|_{x \rightarrow \pm \infty}=0 \\
& \left.w(x)^{\prime}\right|_{x \rightarrow \pm \infty}=0
\end{aligned}
$$

Combining Eq. (3) and Eq. (7) to Eq. (9), the numerical fitting curve of the pipeline settlement $w(x)$ can be calculated by numerical analysis software.

\section{ENGINEERING APPLICATION}

The total length of the tunnel of Zhenpu Road Zhijiang Ocean Park Station of Hangzhou Metro Line 6 is about $2745 \mathrm{~m}$. It is constructed by two earth pressure balance shield machines. The left and right tunnels have the same buried depth. The tunnel diameter is $3.1 \mathrm{~m}$. The twin-tunnel crosses a rigid concrete rainwater pipeline near the Ocean Park Station. The tunnels are $15 \mathrm{~m}$ in depth, and the distance of tunnel axes is $12 \mathrm{~m}$, and the pipeline is 3.2 $\mathrm{m}$ in depth, $1.1 \mathrm{~m}$ in diameter and $1.7 \mathrm{e}^{9} \mathrm{~N} \cdot \mathrm{m}^{2} / \mathrm{m}$ in bending stiffness, and the angle between the tunnel and the pipeline is $75^{\circ}$. The soil layer is mainly silty clay, mucky silty clay containing silt, and according to the thickness-weighted average, the elastic modulus of the soil is $2.4 \mathrm{e}^{7} \mathrm{~Pa}$ and the Poisson's ratio is 0.36 .

The settlement monitoring data of the ground was fitted with the Peck formula as shown in Fig. 2, and the obtained formation loss rate of the ground was $0.75 \%$, and the settlement trough width coefficient was 0.68 .

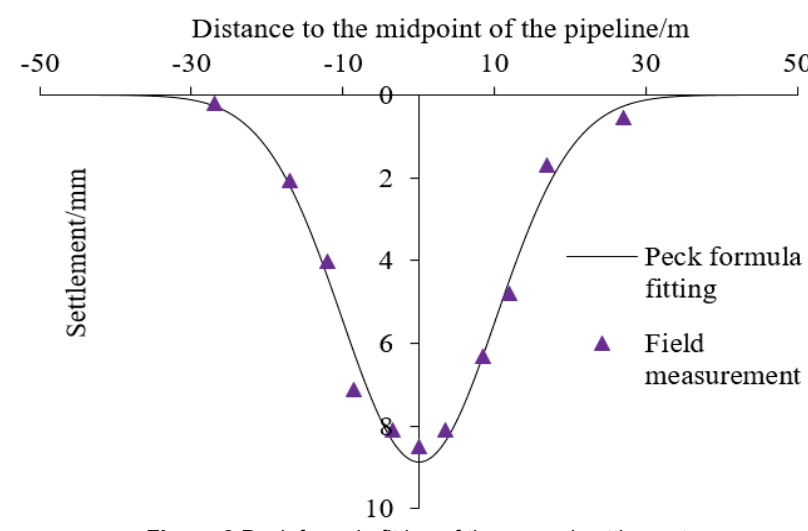

Figure 2 Peck formula fitting of the ground settlement

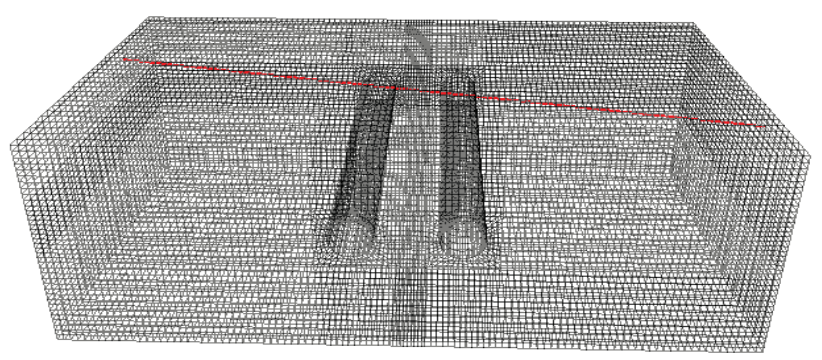

Figure 3 Three-dimensional model

The case was calculated respectively by the above foundation beam method, 3D finite difference program FLAC3D simulation, and field measurement and the obtained results were compared and verified. In the 
numerical simulation, in order to keep consistent with the foundation beam method, the soil was set as linear elastic constitutive model, and the pipeline was simulated by beam element, and the elastic modulus of the beam element was $3 \mathrm{e}^{10} \mathrm{~Pa}$ and the Poisson's ratio was 0.2 . The model size was $100 \times 35 \times 60 \mathrm{~m}$, as shown in Fig. 3 . The bottom of the model was fully constrained, and the side is horizontally constrained, and the top surface is free.

The numerical simulation used the displacement control method based on the soil volume loss rates (DCM) $[15,16]$, in which a non-uniform contraction boundary displacement condition was applied around the tunnel, as shown in Fig. 4. The horizontal and vertical displacement expressions for the tunnel boundary nodes are:

$$
\begin{aligned}
& u_{x}=-\frac{g}{2}(1+\sin \alpha) \cdot \cos \alpha \\
& u_{y}=-\frac{g}{2}(1+\sin \alpha) \cdot \sin \alpha
\end{aligned}
$$

where $g=2 R\left(\sqrt{1+V_{1}}-1\right)$, and $g$ is the gap parameter $(m)$, and $u_{x}, u_{y}$ are the horizontal and vertical displacement of the tunnel boundary.

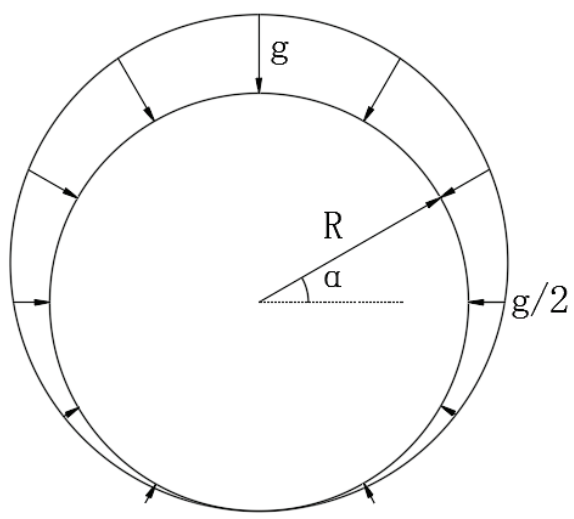

Figure 4 Sectional contraction displacement mode

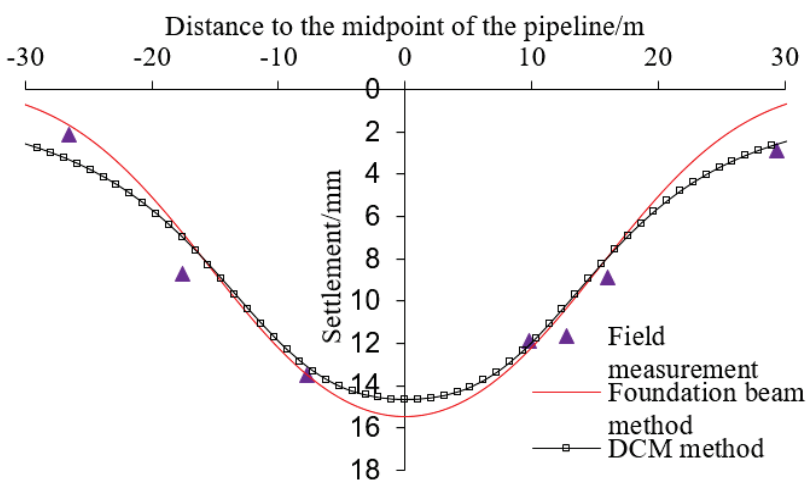

Figure 5 Comparison of the longitudinal displacement curve of the pipeline

Fig. 5 shows the longitudinal displacement curve of the pipeline based on the two-stage foundation beam method, DCM method and field measurement (the position located directly above the middle of the twin-tunnel is the midpoint of the pipeline). It can be seen that there are some differences between the two obtained curves in the foundation beam method and the numerical simulation method, and the latter changes more gently, but the trends of the two are similar, and basically conform to the measured results, which proves the effectiveness of the two-stage foundation beam method.

\section{INFLUENCE FACTORS ANALYSIS}

The settlement of the pipeline caused by tunneling is related to the relative location of the tunnel and pipeline, the design and construction of the tunnel, and the stiffness of the pipeline, such as the angle between the pipeline and the tunnel, the distance between the axes of the twin-tunnel, the soil volume loss rates, and the relative bending stiffness Based on the previous case, the single factor impact analysis was carried out to obtain the settlement curve of the pipeline under different parameters, as shown in Fig. 6 to Fig. 9, where the relative bending stiffness of the pipeline is $\lambda=D / E i^{3} r$ [2]. It can be seen from the figure that the angle between the tunnel and the pipeline has little effect on the settlement of the middle part of the pipeline, and on the whole, the settlement of the pipeline decreases with the increase of the angle. For the spacing $L$ of the tunnel axes, as the $L$ increases, the maximum settlement decreases, and the soil volume loss rate of the twin-tunnel excavation produces a superposition effect on the pipeline. When $L$ is shorter than $20 \mathrm{~m}$, the maximum settlement is located at the midpoint of the pipeline, and if $L$ is longer than $20 \mathrm{~m}$, the superposition effect of the two tunnels' excavation is weakened, and the maximum settlement occurs at the symmetric "double valleys" of the displacement curve. As to the soil volume loss rate, it can be seen that the pipeline settlement value is sensitive to it. Therefore, one of the keys to control the settlement of the pipeline is to control the construction quality of the tunnel and reduce the soil loss. The bending stiffness of the pipeline also has a significant effect on the settlement value of the pipeline. As the relative stiffness of the pipeline decreases, the settlement becomes smaller, and the deformation curve becomes smoother.

\section{Distance to the midpoint of the pipeline $/ \mathrm{m}$}

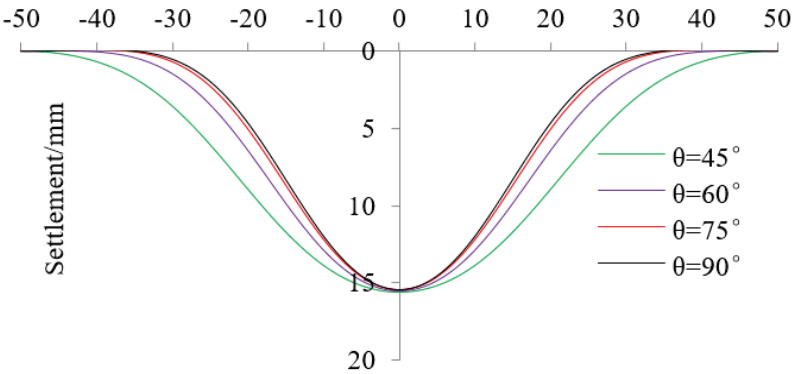

Figure 6 Longitudinal displacement curve of the pipeline with different angles

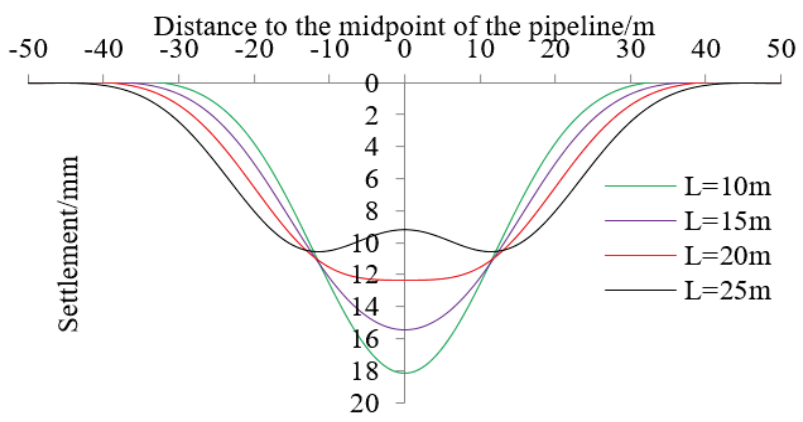

Figure 7 Longitudinal displacement curve of the pipeline with different distance between tunnel axes 
Distance to the midpoint of the pipeline $/ \mathrm{m}$

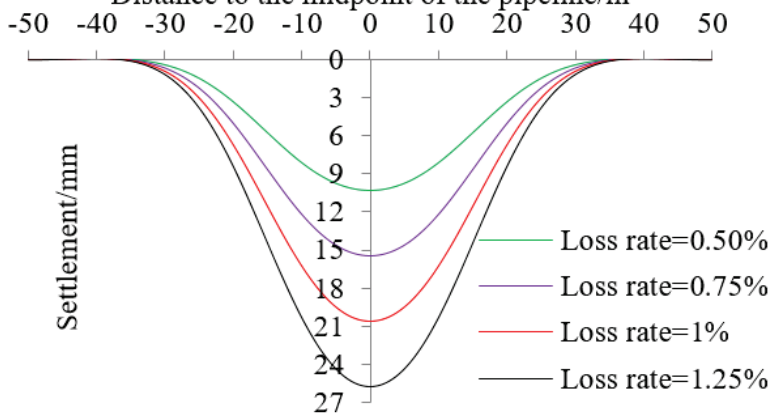

Figure 8 Longitudinal displacement curve of the pipeline with different soil volume loss rates

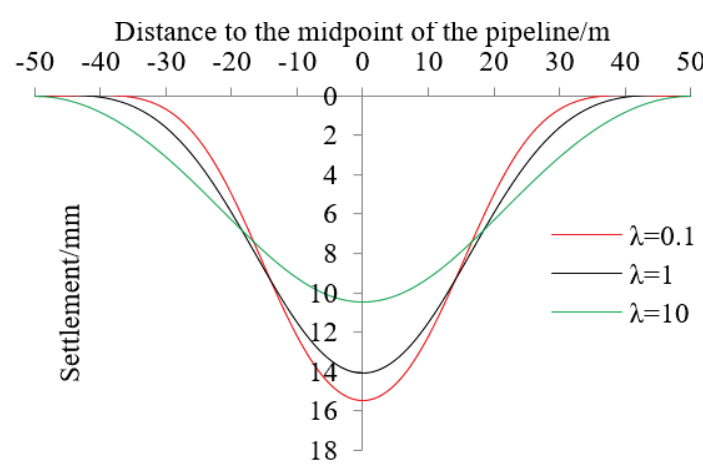

Figure 9 Longitudinal displacement curve of the pipeline with different relative bending stiffness

\section{EFFECT OF SHEARING STIFFNESS ON PIPELINE DEFORMATION}

As above, the pipeline is regarded as the EulerBernoulli beam model, in which only the bending stiffness can be considered while in the Timoshenko beam model, both the bending stiffness and the shearing stiffness of the pipeline can be taken into account. According to the equilibrium differential equation of the displacement and rotation angle, the settlement equation of the Timoshenko beam on the Pasternak foundation can be obtained.

$$
\begin{aligned}
& \left(\frac{2 r G}{C}+1\right) \frac{\mathrm{d}^{4} w}{\mathrm{~d} x^{4}}-\left(\frac{2 r k}{C}+\frac{2 r G}{D}\right) \frac{\mathrm{d}^{2} w}{\mathrm{~d} x^{2}}+\frac{2 r k}{D} w= \\
& =\frac{2 r}{D}\left(k f(x)-G f^{\prime \prime}(x)\right)-\frac{2 r}{C}\left(k f^{\prime \prime}(x)-G f^{(4)}(x)\right)
\end{aligned}
$$

where the shear strength of the pipeline $C=\kappa G_{\mathrm{b}} A$, the shear correction coefficient $\kappa=6\left(1+m^{2}\right)^{2} /\left[7\left(1+m^{2}\right)^{2}+20 m^{2}\right]$

(Cowper correction theory), $\mathrm{m}$ is the ratio of the inner and outer diameter of the pipeline), and $G_{\mathrm{b}}$ is the shear modulus of the pipeline (Pa), and $A$ is the cross-sectional area of the pipeline $\left(\mathrm{m}^{2}\right)$.

Combining Eq. (3), Eq. (8), and Eq. (9), the numerical fitting curve of pipeline settlement can be calculated by the numerical analysis software.

Table 1 Calculation parameters of pipelines
\begin{tabular}{|l|c|c|c|c|}
\hline Category & $\begin{array}{c}\text { Diameter } \\
/ \mathrm{m}\end{array}$ & $\begin{array}{c}\text { Wall } \\
\text { thickness } \\
/ \mathrm{m}\end{array}$ & $\begin{array}{c}\text { Elastic } \\
\text { modulus } \\
/ \mathrm{Pa}\end{array}$ & $\begin{array}{c}\text { Shear } \\
\text { modulus } \\
/ \mathrm{Pa}\end{array}$ \\
\hline Ductile iron pipe & 2.6 & 0.03 & $1.5 \mathrm{e} 11$ & $7.5 \mathrm{e} 10$ \\
\hline Concrete pipe & 3.0 & 0.2 & $3 \mathrm{e} 10$ & $1.2 \mathrm{e} 10$ \\
\hline Steel pipe & 2.5 & 0.02 & $2.06 \mathrm{e} 11$ & $7.9 \mathrm{e} 10$ \\
\hline
\end{tabular}

In order to analyze the influence of the shearing stiffness of the pipeline on the deformation, the common pipelines, such as the ductile iron pipe, the concrete pipe and the steel pipe were taken as the research subjects. Because of the obvious shearing stiffness of the deep beam, the diameter and wall thickness of each pipe was set large, as shown in Tab. 1.

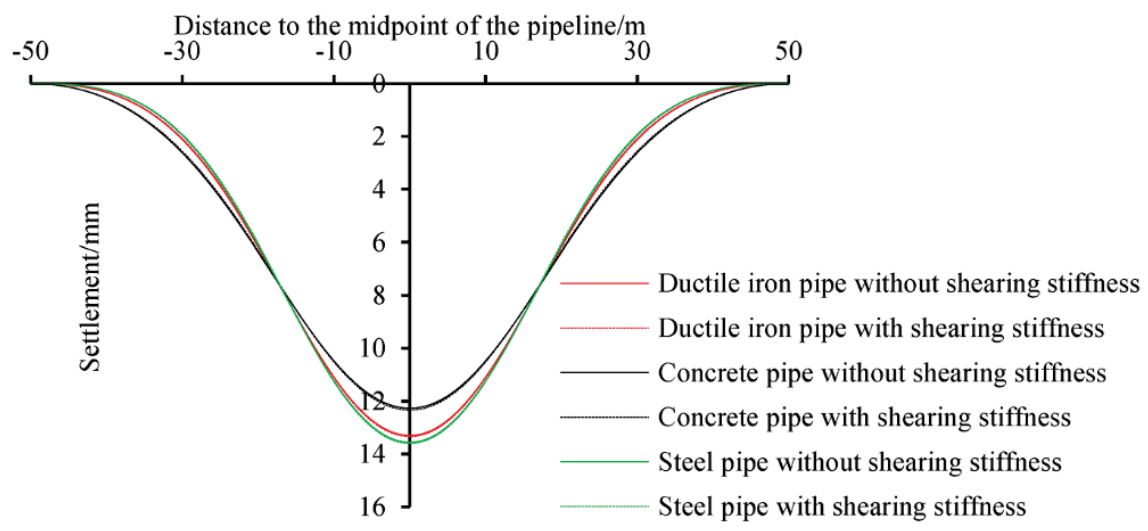

Figure 10 Influence of shearing stiffness on longitudinal displacement of different pipelines

In the deformation analysis of the three types of pipelines, other parameters of pipeline, tunnel and formation are set as above, and as calculated by Eq. (7) and Eq. (12) respectively, the effect of shearing stiffness on the longitudinal deformation of pipelines caused by the tunneling is shown in Fig. 10. It can be seen that for different types of pipelines, the shearing stiffness has little influence on the settlement curve of the pipeline, and the maximum settlement error without consideration of the shearing stiffness does not exceed 1\%. Therefore, for common pipelines, in the calculation of the settlement value caused by tunnel excavation, the impact of the shearing stiffness of pipelines can be ignored.

\section{CONCLUSION}

(1) According to the displacement of the soil caused by the excavation of the twin-tunnel, considering the shear deformation of the soil and the angle between the tunnel and the pipeline, the analysis method of the Pasternak foundation beam for the longitudinal displacement of the pipeline caused by tunnel excavation is established. The calculation result is relatively close to that of the numerical simulation based on displacement controlled method 
(DCM) and the field measured data. This method can be used to estimate the settlement of the continuous rigid pipeline caused by the construction of the underlying twintunnel.

(2) The angle between the tunnel and the pipeline has little effect on the settlement in the middle of the pipeline, and on the whole, the settlement of the pipeline decreases with the increase of the angle. The maximum settlement of the pipeline decreases with the increase of the soil volume loss rate and the relative bending stiffness, and increases with the increase of the spacing of tunnel axes.

(3) For the common pipelines, when calculating the settlement value caused by tunnel excavation, the error without consideration of the shearing stiffness is not more than $1 \%$, and the influence of the shearing stiffness can be ignored. It is reasonable to use the Euler-Bernoulli beam model.

\section{REFERENCES}

[1] Attewell, P. B., Yeates J., \& Selby A. R. (1986). Soil movements induced by tunneling and their effects on pipelines and structures. London: Blackie and Son Ltd.

[2] Klar, A., Vorster, T. E. B., Soga, K., et al. (2005). Soil-pipe interaction due to tunneling: Comparison between Winkler and elastic continuum solutions. Geotechnique, 55(6): 461466. https://doi.org/10.1680/geot.2005.55.6.461

[3] Wei, G., Hu, S., Xing, J. J., et al. (2016). Effect of doubleline parallel shield excavation on adjacent underground pipelines. Journal of Engineering Science and Technology Review, 9(1): 167-173. https://doi.org/10.25103/jestr.091.25

[4] Bai, X. \& Wang, M. (2016). A two-stage method for analyzing the effects of twin tunnel excavation on adjacent tunnels. China Civil Engineering Journal, 49(10), 123-128.

[5] Cheng, K., Yu, F., Liang, R.-Z., Lin, C.-G., Xia, T.-D., \& Xu, R.-Q. (2018). Horizontal deformation of adjacent single pile under tunneling considering shearing effect of piles. Chinese Journal of Geotechnical Engineering, 40(S2), 178-182.

[6] Shi, J., Wang, Y., \& Ng, C. W. W. (2016). Three-dimensional centrifuge modeling of ground and pipeline response to tunnel excavation. Journal of Geotechnical and Geoenvironmental Engineering, 142(11), 04016054. https://doi.org/10.1061/(ASCE)GT.1943-5606.0001529

[7] Zhang, Z., Huang, M., \& Wang, W. (2011). DCBEM-FEM coupling method for response analysis of adjacent pipelines due to tunneling. Chinese Journal of Geotechnical Engineering, 33(10), 1554-1561.

[8] Ma, S., Shao, Y., Liu, Y., et al. (2017). Responses of pipeline to side-by-side twin tunneling at different depths: 3D centrifuge tests and numerical modelling. Tunneling and Underground Space Technology, 66,157-173. https://doi.org/10.1016/j.tust.2017.04.006

[9] Moh, Z. C., Ju, D. H., \& Hwabg, R. N. (1996). Ground movements around tunnels in soft ground. Proceedings of Int Symposium on Geotechnical Aspects of Underground Constructions in Soft Ground. London: A. A. Balkema, 725730.

[10] Mair, R. J., Taylor, R. N., \& Bracegirdle, A. (1993). Subsurface settlement profiles above tunnels in clays. Geotechnique, 43(2): 315-320. https://doi.org/10.1680/geot.1993.43.2.315

[11] Liu, B., Tao, L., Ye, S., et al. (2004). Back analysis prediction system for ground deformation due to subway tunneling excavation. Journal of China University of Mining and Technology, 33(3), 277-282.

[12] Kerr, A. D. (1985). On the determination of foundation model parameters. Journal of Geotechnical Engineering,
111(11), 1334-1340.

https://doi.org/10.1061/(ASCE)0733-9410(1985)111:11(1334)

[13] Morfidis, K. (2003). Research and development of methods for the modeling of foundation structural elements and soil. Thessaloniki: Aristotle University of Thessaloniki.

[14] Xu, L. (2005). Research of the longitudinal settlement of soft soil shield tunnel. Shanghai College of Civil Engineering of Tongji University.

[15] Park, K. H. (2004). Elastic solution for tunneling-induced ground movements in clays. International Journal of Geomechanics, 4(4), 310-318. https://doi.org/10.1061/(ASCE)1532-3641(2004)4:4(310)

[16] Cheng, C. Y., Dasari, G. R., Chow, Y. K., \& Leung, C. F. (2007). Finite element analysis of tunnel-soil-pile interaction using displacement controlled model. Tunneling and Underground Space Technology, 22(4), 450-466. https://doi.org/10.1016/j.tust.2006.08.002

\section{Contact information:}

\section{Jinlong ZHU}

(Corresponding author)

School of Civil Engineering, Hefei University of Technology,

No. 193 Tunxi Road, Hefei, Anhui, 230009, P. R. China

E-mail: zhujinlong_hut@163.com

\section{Dayong ZHU}

Zhejiang University Ningbo Institute of Technology,

No. 1 Qianhu South Road,

Ningbo City, 315100, Zhejiang Province, P. R. China 